\title{
24-Month Effectiveness of Periduoscopic Adhesiolysis in Reducing the Use of Spinal Cord Stimulation in Patient With Chronic Lumbar Pain: A Possible Therapeutic Regimen?
}

Maurizio Marchesini ${ }^{1}$, Marco Baciarello ${ }^{2}$, Roberto Bellacicco 3 , Edoardo Flaviano 4 , Elena G. Bignami

1. Anesthesia and Critical Care, Instituti Clinici Scientifici (ICS) Maugeri, Pavia, ITA 2. II Service Anesthesia, Critical Care and Pain Medicine, Azienda Ospedaliero Universitaria Parma, Parma, ITA 3. II Service Anesthesia, Critical Care and Pain Medicine, Azienda Ospedaliero Univeristaria Parma, Parma, ITA 4. Anesthesia and Critical Care, Papa Giovanni XXIIII, Bergamo, ITA 5. II Service Anesthesia, Critical Care and Pain Medicine, Azienda Ospedaliera Universitaria Parma, Parma, ITA

Corresponding author: Maurizio Marchesini, marchesinidoc@gmail.com

\section{Abstract \\ Objectives}

Epiduroscopy is a minimally invasive procedure that is used in pain therapy to treat lumbar and root pain that is resistant to medical and infiltrative therapies. The indications for periduroscopy are partly shared with those of spinal cord stimulation (SCS): failed back surgery syndrome (FBSS) and stenosis of the vertebral canal in particular. The costs and risks of periduroscopy are considerably lower than those of SCS. The purpose of this study is to evaluate the clinical and economic advantages of integrating periduroscopy as a step prior to SCS for patients with severe lumbar or radicular pain that is unresponsive to pharmacological and infiltrative treatments.

\section{Materials and Methods}

Patients were enrolled if they had FBSS and spinal stenosis with indications for SCS and accepted periduroscopy treatment before the possible SCS trial. Patients were followed up for 24 months with evaluations of clinical data on the day after the procedure and at one and 24 months. The pain trend, satisfaction with the periduroscopy procedure, and the incidence of SCS implants in the study period were analyzed.

\section{Results}

A total of 106 patients were enrolled. Immediately after the procedure and in the first month, the reduction of pain and the level of patient satisfaction were high, but they were drastically reduced at 24 months with a progressive reappearance of symptoms that substantially overlapped with the pre-surgery levels. At 24 months, $48 \%$ of the patients underwent a neurostimulation trial, and a significant percentage of them were able to avoid the implantation of an SCS.

Review began 07/02/2021 Review ended 08/16/2021 Published 08/30/2021

\section{() Copyright 2021}

Marchesini et al. This is an open access article distributed under the terms of the Creative Commons Attribution License CC-BY 4.0., which permits unrestricted use, distribution, and reproduction in any medium, provided the original author and source are credited.

\section{Conclusions}

Periduroscopy appears to be rational as a step prior to SCS in terms of the improvement of pain symptoms in the short term, the definitive results in a significant percentage of patients, and the significant economic savings for the health system.

Categories: Anesthesiology, Pain Management

Keywords: periduroscopy, spinal cord stimulation, chronic pain, hta, chronic post surgical pain

\section{Introduction}

The story of epiduroscopy is one of intuition [1]. It is the same kind of intuition that is shared with the great seafarers who profoundly changed our way of thinking about the world in the XV century and moved the borders of the known universe a bit forward. But in this case, the "New World" is the features of the tiny space known as the epidural space. But this anatomical space has been well known over the centuries, so the attribute "new" is not suitable. What is really "new" is the insight that allows us to think of it as navigable and explorable, regardless of the size (the normal diameter ranges are between 15 and $27 \mathrm{~mm}$ for the vertebral canal and between 20 and $23 \mathrm{~mm}$ for the intervertebral foramen) [2].

The first endoscopic study of the spinal canal was made using arthroscopic equipment and vertebral columns removed from a cadaver, dating back to Burman [3] in the early 1930s. After the first reports, the technique was no longer mentioned for decades until 1967 [4]. Only by the end of 1980 has it been primarily 
used for examining the epidural space thanks to the development of small-caliber flexible optics [5]. Since then, many other improvements have been made, and the interest has never receded; at last, the power of that original intuition came across to technological progress.

Epiduroscopy is a minimally invasive technique that is useful for assessing the epidural space through a flexible instrument connected to an optical fiber. In addition to simple clinical evaluation, epiduroscopy allows for treatments such as the targeted delivery of drugs, debridement of adhesions, and reduction of inflammatory factors with copious lavage [6-7]. Even in non-operated patients (so-called "virgin back"), epiduroscopy can be a valuable approach for possible interventions on anatomical structures that can be pain generators in pathological conditions [8]. However, "minimally invasive" does not mean "free of complications." Transient neurological symptoms (TNS) and post-procedural pain in the affected area are the most frequent and benign manifestations. Loss of acuity, encephalopathy resulting in rhabdomyolysis, neurological bladder, seizures, and pneumocephalus are among the other complications worth mentioning because of their potential severe and permanent consequences, although they are much less common [9].

Spinal cord stimulation (SCS) was first described and applied by Shealy [10] in the late 1960s and has become a widely used and effective alternative for the management of chronic refractory pain that is unresponsive to conservative therapies [11-13] even in elderly patients [14]. Although the clinical benefit of SCS is substantial, detailed knowledge of how SCS inhibits pain is lacking. The technique acts both at the spinal [15] (mostly) and the supraspinal [16] levels, depending on the type of stimulation pattern. It is believed to work according to gate control theory [17] by stimulating the large-size axons (A-beta fibers) [18] and interneurons in the dorsal roots, thus decreasing the influx of painful stimuli.

SCS may evoke the release of serotonin and norepinephrine into the dorsal horn from descending fibers originating in the supraspinal pain-modulatory structures [19]. Other changes in the neurochemical environment at the spinal level are related to the local concentrations of gamma-aminobutyric acid (GABA) and acetylcholine (ACh) [20]. Interestingly, this technology does not only have immediate action; its positive effects have been revealed to be long-lasting because of neuronal plasticity [21].

The most common complication in SCS is hardware problems, which include electrode migrations and lead fracture [22]. The most significant complications are associated with neurological damage due to intraoperative root or spinal cord injury, epidural hematoma, and infections (particularly epidural abscess) [23]. Notably painful stimulation and inadequate coverage of the painful area sometimes necessitate either repositioning or removing the electrode [24]. In 1998, it was estimated that SCS was applied to about 15000 patients annually worldwide, of which about 5000 were cases in Europe [25]. Nowadays, the number has at least doubled.

According to the "Pain in Europe" study [26], up to 19\% of the whole European population suffers from moderate or severe back pain. In Italy. this percentage is even higher reaching $26 \%$. Back pain has profound consequences on people's lives and incurs tremendous costs for society since most individuals are working age. What we are facing here is a healthcare emergency, and the right tool needs to be used for the right candidate to avoid further waste of time and economic resources.

The aim of this study is to determine whether epiduroscopy is capable of playing a significant role in the treatment regimen of severe lumbar pain that does not respond to standard medical therapies.

\section{Materials And Methods}

This study was approved by the responsible ethics committee, and informed consent was acquired from all patients. We enrolled 106 patients with failed back surgery syndrome (FBSS) and stenosis of the canal. Patients were enrolled with the following inclusion criteria:

- Age 18-85 years
- Patients with FBSS and spinal stenosis
- Able to sign an informed consent form

The following exclusion criteria were applied:

- Patients with clinically unstable disease (all morbid forms whose treatment is not stable over time but requires continuous pharmacological and dosage adjustments or require further investigations)

- Patients who have been diagnosed with severe neuropsychiatric disease and have received pharmacological treatment under constant medical supervision for which there is a contraindication for invasive treatments 


\title{
Cureus
}

\author{
- History of vertebral fractures \\ - Tumors or infections affecting the spine \\ - Visual impairment (glaucoma, diabetic retinopathy) \\ - Chronic primary or secondary headache \\ - Pregnancy \\ - Coagulopathies (INR > 1. 5)
}

\section{Analgesic visit and follow-up}

The patients underwent an antalgic visit before admission with an evaluation of the blood count, coagulation, ECG, and anesthesiological examination. Pain characteristics were assessed before the periduroscopic procedure, immediately after the procedure, at one month, and at 24 months using a questionnaire, in which the Numeric Rating Scale (NRS) and Global Perceived Effect (GPE) indices were analyzed. The NRS value was calculated by asking the patients about their minimum, average, and maximum intensity of pain during the day on a numerical scale of one to 10. The GPE value was calculated by asking patients to rate their pain relief compared to baseline as $>50 \%$, between 30 and $50 \%$, and $<30 \%$. The costs incurred by the National Health Service (NHS) for the periduroscopic adhesiolysis procedure (LOA) and SCS were analyzed (costs in 2016-2018).

\section{Periduroscopy}

All the procedures were performed by a single operator who had experience with more than 80 procedures. The procedures were performed with the Resascope System (AMS group srl, Padua, Italy), which is composed of a 10F adjustable epidural video-guided catheter with an external diameter of $3.3 \mathrm{~mm}$, a length of $30 \mathrm{~cm}$, and two channels. One channel is for saline infusion for washing the epidural space, thus improving visibility, and the other one is for the collection of the fluid. Three entry channels are used for the insertion of flexible fiber optics and other tools and have an internal diameter of $1.25 \mathrm{~mm}$.

\section{Results}

\section{Demographics}

We recruited 106 patients (41 men and 65 women) who underwent periduroscopic adhesiolysis and were diagnosed with FBSS (92 pcs) or canal stenosis (14 pcs). The median age was 57 years (range $30-80$ years).

\section{Adverse events, screening failure, and dropout}

During the procedure, three adverse events that led to stopping the procedure were recorded: retinal hemorrhage, significant peridural bleeding, and hemodynamic instability. During the first month, three patients were excluded from the study due to no longer fulfilling the inclusion and exclusion criteria. It was not possible to administer the questionnaire to six patients after the procedure, to 11 patients at the followup at one month, and to 25 patients at the follow-up at 24 months. Thus, there were 58 patients in the study at 24 months (Figure 1). 


\section{Cureus}

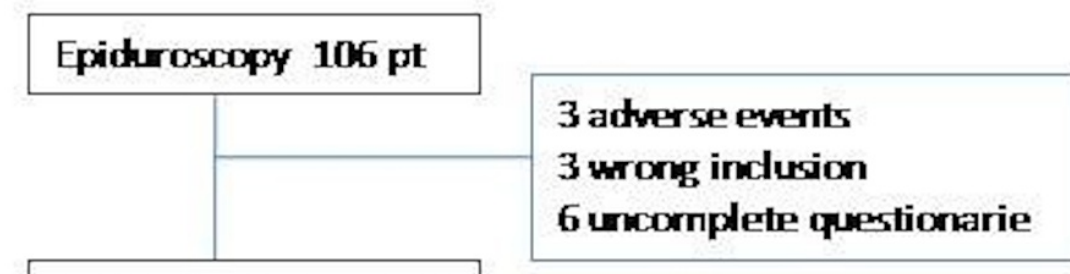

\section{Post Porcedure 100 pt}

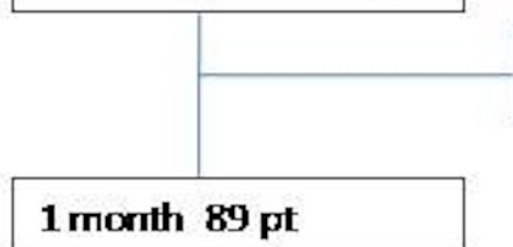

\section{1 drop out}

\section{1 monith 89 pt}

\section{5 drop out}

24 moniths 58 pt

FIGURE 1: Patients' screening

\section{NRS and GPE evaluation}

The pain tendencies were similar for the maximum, average, and minimum NRS. There was a significant reduction after the procedure, a relative rise at one month, and a substantial return to near the baseline value at 24 months. The values and statistical analysis results are summarized in Table 1.

\begin{tabular}{|c|c|c|c|c|c|c|c|}
\hline & Baseline & $\begin{array}{l}\text { After } \\
\text { procedure }\end{array}$ & $\begin{array}{l}1 \\
\text { month }\end{array}$ & $\begin{array}{l}24 \\
\text { months }\end{array}$ & $\begin{array}{l}\text { Baseline vs. after } \\
\text { procedure }\end{array}$ & $\begin{array}{l}\text { Baseline vs. } 1 \\
\text { month }\end{array}$ & $\begin{array}{l}\text { Baseline vs. } 24 \\
\text { months }\end{array}$ \\
\hline NRS Max & 8.8 & 4.2 & 6.2 & 7.2 & $\mathrm{p}<0.001$ & $p<0.01$ & n. s. \\
\hline $\begin{array}{l}\text { NRS } \\
\text { Average }\end{array}$ & 6.7 & 3.9 & 5.4 & 6.5 & $\mathrm{p}<0.001$ & $p<0.01$ & n.s. \\
\hline NRS Min & 3 & 1.2 & 2.1 & 2.4 & $p<0.001$ & n. s. & n. s. \\
\hline
\end{tabular}

TABLE 1: Pain relief after periduroscopy

NRS: Numeric Pain Rating Scale

After the procedure, GPE was $>50 \%$ for 45 patients, $30-50 \%$ for 24 patients, and $<30 \%$ for 25 patients. At the one-month follow-up, GPE was $>50 \%$ for 27 patients, $30-50 \%$ for 14 patients, and $<30 \%$ for 48 patients. At 24 months, GPE was $>50 \%$ for 17 patients, 30-50\% for six patients, and $<30 \%$ for 35 patients (Figure 2). At the 24-month follow-up, 28 patients started the process of spinal cord stimulator implantation due to insufficient analgesic coverage of periduroscopic adhesiolysis. Of these, two patients failed the trial, and eight complained of ineffectiveness, which led to the removal of the implant in four cases. Of the 28 patients who started the implantation procedure, 19 started with a post-periduroscopic GPE $<30 \%$, five had $30-50 \%$, and four had $>50 \%$. 


\section{Cureus}

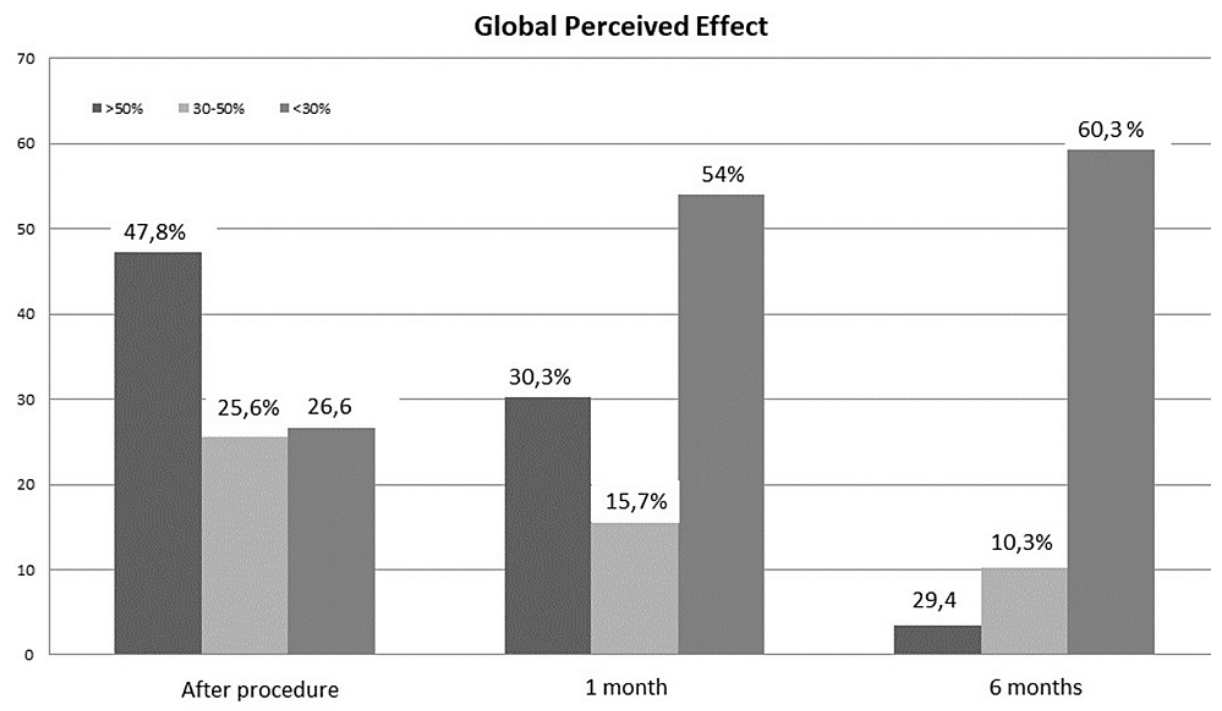

FIGURE 2: Global Perceived Effect

\section{Cost evaluation of epiduroscopy vs. SCS}

The total cost of periduroscopic adhesiolysis is $€ 2,472$ per patient. The total cost of the SCS trial and final interventions is at least $€ 13,398$ per patient. The costs for each component are shown in Table 2.

\begin{tabular}{|c|c|c|}
\hline Cost element & Epiduroscopy & Spinal cord stimulation \\
\hline Clinical preoperative evaluation (with ECG and blood exam) & $79.2 €$ & $79.2 €$ \\
\hline Operating room & $598 €$ & $650 €$ \\
\hline Personnel & $76 €$ & $300 €$ \\
\hline Instruments & $1.350 €$ & $12.000 €$ \\
\hline Imaging & $19.5 €$ & $19.5 €$ \\
\hline Hospitalization & $350 €$ & $350 €$ \\
\hline TOTAL & $2.472 €$ & $13.398 € €$ \\
\hline
\end{tabular}

TABLE 2: Costs for procedure in Italian Health System

\section{Discussion}

In the 106 patients analyzed, the periduroscopic adhesiolysis procedure was particularly effective immediately after the procedure and at the one-month follow-up. The pain relief showed a significant difference between baseline and immediately after the procedure, and this benefit persisted for the first month with a progressive return to the original level during the next 24 months (Figure 2). The GPE variation showed an excellent and modest outcome (> 50\% and $30-50 \%$ ) in $73.4 \%$ of patients immediately after the procedure and $58 \%$ at the one-month follow-up.

In the 24-month follow-up, the procedure tended to lose efficacy for patients, with optimal and intermediate outcomes occurring in $39.7 \%$, as well as a change of 0.2 in NRS compared to the baseline. This result seems to agree with a recent review of the literature showing a progressively diminishing benefit over time [27]. Interestingly, the absence of pain reduction from baseline was not fully related to the GPE evaluation, showing how pain is not the only aspect that the patients consider in their outcomes. In the 58 patients analyzed at the 24-month follow-up, 28 (48\%) started the procedure of SCS.

All 106 patients enrolled at the start of the study had potential indications for both SCS and epiduroscopy, but only 24 (41\%) of the 58 patients analyzed at 24 months benefited from the SCS implant. The data seem to 
suggest that the periduroscopic adhesiolysis procedure tends to be an effective intermediate treatment to distinguish cases of pain mixed with a more significant inflammatory component, where epiduroscopy would be more effective, as well as cases of mixed pain with a more significant neuropathic component, where SCS is the only effective therapeutic option.

The cost per patient of the two procedures is significantly higher for SCS ( $€ 13,398 /$ pt) than epiduroscopy ( $€$ $2,472 / \mathrm{pt}$ ) due to the high cost of the internal pulse generator (IPG) and device with the same length of hospitalization. Considering the 24-month follow-up with 58 patients, a therapeutic procedure that provides epiduroscopy plus the SCS implanted $(€ 464,928)$ would be more economical than a procedure that provides for the preventive implantation of SCS for all patients without epiduroscopy attempt before (€ 770,084), with a total saving of $€ 305.106$ (corresponding to $€ 5,261 / p t$ ).

The critical number of dropouts poses limitations to our study at 24 months and caused a substantial dispersion of data. We cannot say whether the dropout patients sought new surgical interventions or other medical therapy. As the primary center for the region, we can most likely affirm that those patients did not move to other pain centers for SCS implantation, but we cannot be completely sure. Further studies are needed to identify predictive factors that can predict which of the two methods will be more effective and to evaluate what other treatments will be effective after 24 months in patients who do not undergo spinal cord stimulation and have a lower NRS and GPE at baseline.

\section{Conclusions}

The use of periduroscopic adhesiolysis as an intermediate procedure before SCS and can be included in the treatment regimen of severe lumbar pain that is refractory to pharmacological treatments and to reduce the use of SCS implants to save resources for the NHS. The analgesic relief of the periduroscopic procedure tends to lose efficacy after 24 months, but an increase in SCS implants does not accompany this trend. Further studies are needed to determine predictive factors that are capable of predicting the best treatment for the clinical needs of individual patients.

\section{Additional Information \\ Disclosures}

Human subjects: Consent was obtained or waived by all participants in this study. Ethics Committee Università di Parma issued approval 13479. Research involving human subject complied will all relevant regulation, institutional policies and is in accordance with the tenets of Helsinki Declaration (as amended in 2013). Copies of ethical approval were provided to the European Commission Before initiating the study. The specific WP has been approved by Ethical Committee of University of Parma 26 feb 2014. Animal subjects: All authors have confirmed that this study did not involve animal subjects or tissue. Conflicts of interest: In compliance with the ICMJE uniform disclosure form, all authors declare the following: Payment/services info: Perioduroscopy procedures were partially funded by FP7 grant number 602736, the Pain Omic (WP) FP7 project, the follow-up of the patients, and the subsequent implantation of the medullary stimulator without the use of funds. Financial relationships: All authors have declared that they have no financial relationships at present or within the previous three years with any organizations that might have an interest in the submitted work. Other relationships: All authors have declared that there are no other relationships or activities that could appear to have influenced the submitted work.

\section{References}

1. Blomberg R: A method for epiduroscopy and spinaloscopy. Presentation of preliminary results . Acta Anaesthesiol Scand. 1985, 29:113-6. 10.1111/j.1399-6576.1985.tb02169.x

2. Watts R: Lumbar vertebral canal size in adults and children: observations from a skeletal sample from London, England. Homo. 2013, 64:120-8. 10.1016/j.jchb.2013.01.002

3. Raffaeli W, Righetti D, Andruccioli J, Sarti D: Periduroscopy: general review of clinical features and development of operative models. Acta Neurochir Suppl. 2011, 108:55-65. 10.1007/978-3-211-99370-5 10

4. Ooi Y, Satoh Y, Inoue K, Mikanagi K, Morisaki N: Myeloscopy, with special reference to blood flow changes in the cauda equina during Lasègue's test. Int Orthop. 1981, 4:307-11. 10.1007/BF00266074

5. Shimoji K, Fujioka H, Onodera M, Hokari T, Fukuda S, Fujiwara N, Hatori T: Observation of spinal canal and cisternae with the newly developed small-diameter, flexible fiberscopes. Anesthesiology. 1991, 75:341-4. 10.1097/00000542-199108000-00024

6. Bosscher HA, Heavner JE: Diagnosis of the vertebral level from which low back or leg pain originates. A comparison of clinical evaluation, MRI and epiduroscopy. Pain Pract. 2012, 12:506-12. 10.1111/j.15332500.2012.00549.x

7. Rabinovitch DL, Peliowski A, Furlan AD: Influence of lumbar epidural injection volume on pain relief for radicular leg pain and/or low back pain. Spine J. 2009, 9:509-17. 10.1016/j.spinee.2009.03.003

8. Umeh R, Fisahn C, Burgess B, Iwanaga J, Moisi M, Oskouian RJ, Tubbs RS: Transforaminal ligaments of the lumbar spine: a comprehensive review. Cureus. 2016, 8:e811. 10.7759/cureus.811

9. Marchesini M, Flaviano E, Bellini V, Baciarello M, Bignami EG: Complication of epiduroscopy: a brief review and case report. Korean J Pain. 2018, 31:296-304. 10.3344/kjp.2018.31.4.296

10. Shealy CN, Mortimer JT, Reswick KB: Electrical inhibition of pain by stimulation of dorsal column: preliminary clinical reports. Anesth Analg. 1967, 4:489-91. 
11. Van Buyten JP, Van Zundert J, Vueghs P, Vanduffel L: Efficacy of spinal cord stimulation: 10 years of experience in a pain centre in Belgium. Eur J Pain. 2001, 5:299-307. 10.1053/eujp.2001.0249

12. Kumar K, Malik S, Demeria D: Treatment of chronic pain with spinal cord stimulation versus alternative therapies: cost-effectiveness analysis. Neurosurgery. 2002, 51:106-15. 10.1097/00006123-200207000-00016

13. Hallo A, Martínez H, Jácome-Calderón KE, Rodríguez M: Spinal cord stimulation: viable therapeutic option for postlaminectomy syndrome in elderly patients. Cureus. 2021, 13:e15675. 10.7759/cureus.15675

14. Granville M, Berti AF, Jacobson RE: Use of spinal cord stimulation in elderly patients with multi-factorial chronic lumbar and non-radicular lower extremity pain. Cureus. 2017, 9:e1855. 10.7759/cureus.1855

15. de Andrade DC, Bendib B, Hattou M, Keravel Y, Nguyen JP, Lefaucheur JP: Neurophysiological assessment of spinal cord stimulation in failed back surgery syndrome. Pain. 2010, 150:485-91. 10.1016/j.pain.2010.06.001

16. Stancák A, Kozák J, Vrba I, Tintera J, Vrána J, Polácek H, Stancák M: Functional magnetic resonance imaging of cerebral activation during spinal cord stimulation in failed back surgery syndrome patients. Eur J Pain. 2008, 12:137-48. 10.1016/j.ejpain.2007.03.003

17. Melzack R, Wall PD: Pain mechanisms: a new theory . Science. 1965, 150:971-9. 10.1126/science.150.3699.971

18. Holsheimer J: Computer modelling of spinal cord stimulation and its contribution to therapeutic efficacy . Spinal Cord. 1998, 36:531-40. 10.1038/sj.sc.3100717

19. Song Z, Ultenius C, Meyerson BA, Linderoth B: Pain relief by spinal cord stimulation involves serotonergic mechanisms: an experimental study in a rat model of mononeuropathy. Pain. 2009, 147:241-8. 10.1016/j.pain.2009.09.020

20. Barchini J, Tchachaghian S, Shamaa F, et al.: Spinal segmental and supraspinal mechanisms underlying the pain-relieving effects of spinal cord stimulation: an experimental study in a rat model of neuropathy. Neuroscience. 2012, 215:196-208. 10.1016/j.neuroscience.2012.04.057

21. Lu Y, Perl ER: A specific inhibitory pathway between substantia gelatinosa neurons receiving direct C-fiber input. J Neurosci. 2003, 23:8752-8. 10.1523/JNEUROSCI.23-25-08752.2003

22. Tanaka S, Barron KW, Chandler MJ, Linderoth B, Foreman RD: Low intensity spinal cord stimulation may induce cutaneous vasodilation via CGRP release. Brain Res. 2001, 896:183-7. 10.1016/s0006-8993(01)021448

23. L Manchikanti, S Abdi, S Atluri, et al.: An update of comprehensive evidence-based guidelines for interventional techniques in chronic spinal pain. Part II: guidance and recommendations. Pain Physician. 2013, 16:49-283.

24. Patel SK, Gozal YM, Saleh MS, Gibson JL, Karsy M, Mandybur GT: Spinal cord stimulation failure: evaluation of factors underlying hardware explantation. J Neurosurg Spine. 2019, 4:1-6. 10.3171/2019.6.SPINE181099

25. Baber Z, Erdek MA: Failed back surgery syndrome: current perspectives. J Pain Res. 2016, 9:979-87. 10.2147/JPR.S92776

26. Breivik H, Collett B, Ventafridda V, Cohen R, Gallacher D: Survey of chronic pain in Europe: prevalence, impact on daily life, and treatment. Eur J Pain. 2006, 10:287-333. 10.1016/j.ejpain.2005.06.009

27. Geudeke MW, Krediet AC, Bilecen S, Huygen FJ, Rijsdijk M: Effectiveness of epiduroscopy for patients with failed back surgery syndrome: a systematic review and meta-analysis. Pain Pract. 2021, 21:468-81. 10.1111/papr.12974 